\title{
Automated Peripheral Neuropathy Assessment using Optical Imaging and Foot Anthropometry
}

\author{
Hafeez-U. R. Siddiqui*, Student Member IEEE, Michelle Spruce, Stephen Alty, Senior Member IEEE \\ and Sandra Dudley*, Member, IEEE
}

\begin{abstract}
A large proportion of individuals who live with type-2 diabetes suffer from plantar sensory neuropathy. Regular testing and assessment for the condition is required to avoid ulceration or other damage to patient's feet. Currently accepted practice involves a trained clinician testing a patient's feet manually with a hand-held nylon monofilament probe. The procedure is timeconsuming, labor-intensive, requires special training, is prone to error and repeatability is difficult. With the vast increase in type2 diabetes, the number of plantar sensory neuropathy sufferers has already grown to such an extent as to make a traditional manual test problematic. This paper presents the first investigation of a novel approach to automatically identify the pressure points on a given patient's foot for the examination of sensory neuropathy via optical image processing incorporating plantar anthropometry. The method automatically selects suitable test points on the plantar surface that correspond to those repeatedly chosen by a trained podiatrist. The proposed system automatically identifies the specific pressure points at different locations, namely the toe (hallux), metatarsal heads and heel (Calcaneum) areas. The approach is generic and has shown $100 \%$ reliability on the available database used. The database consists of Chinese, Asian, African and Caucasian foot images.
\end{abstract}

Index Terms - Optical image processing, plantar surface, peripheral sensory neuropathy (PSN), diabetes, foot anthropometry, binary image, contours, polygon, skin tone, hue saturation voluminous (HSV), red, green blue (RGB).

\section{INTRODUCTION}

$\mathrm{D}$ IABETES mellitus (DM) is a chronic illness affecting more than $8 \%$ of the developed world, with growing numbers also in countries such as China and India. Diabetic peripheral sensory neuropathy (PSN) is a common complication of DM, with typically $60 \%$ of all type-2 diabetics developing this condition within 10 years of being first diagnosed. With the enormous growth of type-2 diabetes the frequency of diabetic neuropathies is noticeably increasing, but actual numbers remain undisclosed, with variable reports on diabetic patients depending mostly on the

Manuscript received October 21st 2014; revised January $19^{\text {th }} 2015$; accepted February $14^{\text {th }} 2015$. This work was supported by London South Bank University, 103 Borough Road, London, SE1 0AA, U.K. Asterisks indicate corresponding authors.

Hafeez-U. R. Siddiqui*, Stephen R. Alty and Sandra Dudley* are with the School of Engineering, London South Bank University, 103 Borough Road, London, SE1 0AA, U.K. (e-mail: siddiqh3, steve.alty and dudleyms@1sbu.ac.uk).

Michelle Spruce is an honorary research fellow in the School of Health and Social Care, London South Bank University. (E-mail:m.spruce@1sbu.ac.uk). criteria and methods used to identify neuropathy [1]. Roughly 27 million diabetics are registered in Europe alone. The number of PSN sufferers has already grown to such an extent as to make a traditional manual PSN test challenging. This paper presents the first investigation of a novel approach to automatically identify the pressure points on a given patient's foot for the examination of PSN via optical image processing.

In the United Kingdom there are presently around 3.8 million people living with diabetes and the current cost of direct diabetic patient care (treatment, intervention and complications assistance) is estimated at $£ 9.8$ billion [1], [2]. This number is expected to increase to 6.25 million by $2035 / 6$. This projected growth is likely to have significant impact on National Health Service (NHS) resources. Of course this is not solely a UK problem, but internationally one can then appreciate the vast issue.

One side effect of type-2 diabetes is PSN and this affects the peripheral extremities of the body, often initialising with numbness of the toes and subsequently extending to the plantar aspect of the feet. PSN is commonly regarded as a key factor in the development of ulceration, thus its early diagnosis and correct assessment are important factors to manage this condition and identify higher risk patients. Sufferers lose mobility and this degenerative process has a dramatic impact on their quality of life. If not carefully monitored and timely treated, lower limb amputation is unfortunately a common consequence [3]. The maintenance and appreciation of healthy feet is fundamental in enabling sufferers to preserve an active and healthy lifestyle and reduce sufferers' risk of ulceration. One of the main causes of ulceration is diabetic PSN [4]. Foot complications are indeed, some of the most frequent problems of diabetic mellitus, a key contributor to medical costs, as $50 \%$ of all inpatient admissions of diabetes are due to foot disease [5].

Hitherto the Semmes-Weinstein Monofilament Examination (SWME) method is one of the most common tests used to identify PSN and increased risk of ulceration, through the examination of five pressure points at specific weight bearing areas, namely the toe (hallux), metatarsal and heel (Calcaneum). An extruded homopolymer monofilament (SWM) probe is applied by a trained clinician. The SWM is designed to bend by $10 \mathrm{~mm}$ (gauged commonly by sight) when $10 \mathrm{~g}(98 \mathrm{mN})$ force is applied. Studies have shown that the inability to detect the SWM, when it bends by $10 \mathrm{~mm}$ at $10 \mathrm{~g}$ force, indicates a degree of neuropathy. The rationale for this is based upon world health organization (WHO) and National institute for health and care excellence (NICE) guidelines which indicate that reduced sensation to a high pressure site is 
an accepted risk factor for the development of ulceration [6]. Currently, professionals rely on subjective judgment as to which areas may constitute a high pressure site; this is frequently based upon bony prominences, deformity or soft tissue indicators. On this basis it is the gold standard that all these identified areas are then tested for PSN (a risk factor for the development of ulceration). The SWME, although the most widely used, is considered cumbersome and laborintensive. Repeatability is difficult to maintain and testing can be prone to experimenter bias. Moreover, this issue may be amplified when a patient is seen by a different practitioner on each visit [7].

Ultimately, numbers suffering from type-2 diabetes has already reached such rates as to make a traditional PSN test unfeasible. Furthermore, the resources required to test current sufferers outstrip those provided by the UK NHS. Thus, there is an imperative need to develop an automated system that is, autonomous, repeatable, and simplifies the testing procedure, thus enabling tests to be performed within medical drop-in centers and general practices but also, for example, in locally based pharmacies. Additionally, a system that provides resultant photographic evidence of patients' feet and their condition over time is valuable to both medical practitioners and researchers. Automated techniques such as that presented here would reduce waiting times, increase the number of patients that can be tested and provide a neutral, unbiased selection of test points.

We describe a novel approach to automatically select suitable pressure points, namely toe (hallux), metatarsal heads and heel (Calcaneum), on a given patient's foot via optical image processing incorporating plantar anthropometry i.e. automating the accepted SWME. Importantly, these pressure points correspond to those chosen by a trained podiatrist for PSN examination. The system is embedded with in-house designed software which ultimately produces an image of the tested foot and the five pressure points detected. These results are then sent to the health care provider via email.

The human foot is a complex structure, playing an important role in the locomotion processes of the lower extremity [8]. People vary in foot shape. Gender, age, race, and even lifestyle habits play an important role in shaping the foot [9]. Foot anthropometry is the measurement of the size and proportion of different parameters of the foot and is accepted in studies related to ergonomics, forensics science and anthropology. The most common foot parameters used for foot anthropometry are foot length, ball length, ball width, girth, heel width, instep height, instep width and toe height $[10,11]$.

Previous anthropometric studies of foot shape have used differing protocols and measurement devices. Most anthropometric approaches directly measure the foot dimensions using a Vernier caliper or cloth tape flat ruler, whilst others have used foot prints. Additional studies have devised physical foot measurement platforms [10]. There are also methods devised to quantify foot posture or mobility from digital images; these methods have a commonality in that they involve capturing a digital image of the foot. However, the post-processing procedures differ amongst them [12, 13]. Though their research objectives are diverse, the methods followed by those referenced involved manual intervention such as marking "bony" land marks, painting the plantar

surface with "face and body" paint and attached retro reflective targets [14], making the procedures cumbersome and difficult to repeat, obviously unsuitable as an automated SWME replacement method.

Previous work presented by the authors [15] covered pressure point detection by plantar contact area using optical imaging. An optical scanning technique obtained the human plantar surface and specific skin color space was used to detect the plantar contact area, but only Caucasian and nonflat feet patient groups were considered. Here, an improved imaging method to automatically identify suitable pressure points along the plantar surface is proposed using the same physical system architecture as described in [15]. Furthermore, an improved algorithm is presented that extracts plantar surface pressure points via a combined optical image processing and plantar anthropometry approach independently of patient's race.

\section{Methodology}

The authors concentrate here on the optical image detection and processing of a patient's foot. In order to reflect the accepted SWME method the footrest again is a perforated sheet enabling a mechanically driven $10 \mathrm{~g}(98 \mathrm{mN})$ probe to be admitted to the patient's foot through the perforations once the pressure points have been correctly identified via the optical imaging method described. This mechanical probe and optical integration will be presented elsewhere. To assist the supervisory development of the program, a fixed small and soft stud is introduced to the underside of the perforated sheet as shown in Fig. 1(d).

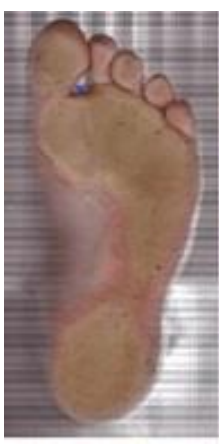

(a)

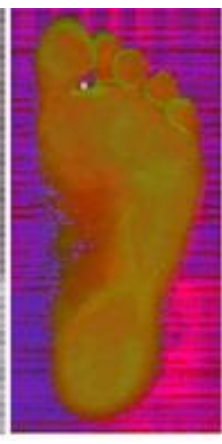

(b)

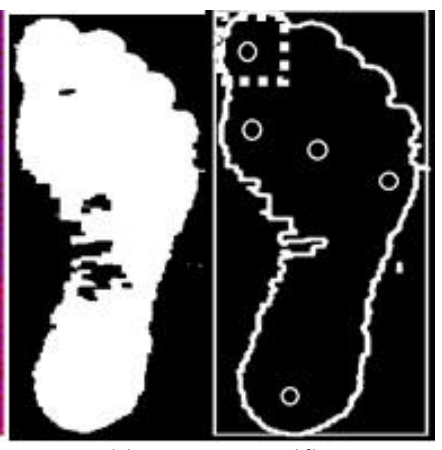

(c)

(d)
Fig. 1. Processing input image (a): Plantar image in RGB color space. (b): Plantar image in HSV space (c): Binary Image. (d): Outer boundary of detected plantar surface

The stud is known as a foot stopper (FS) and has two main purposes:

1) It assists patients to position their foot correctly on the perforated sheet.

2) It acts as a reference point for the algorithm used to perform the foot anthropometry calculations.

The blue color of the FS helps in its identification during the image processing of the plantar surface in terms of spatial coordinates. The foot is scanned and the foot image is obtained in Red, Green and Blue (RGB) color space as shown in Fig. 1(a). RGB is an additive color system, where primary colors are combined in different quantities to form other 
colors. In RGB space a color is described by indicating the amount of red, green, and blue color. When all colors have the maximum value, the resulting color is white and black vice versa [16]. In RGB color space the luminance information is embedded into each band in the visible light spectrum of the image. Varying levels of brightness in an image causes RGB values to shift. The primary color components are highly correlated and therefore it is difficult to execute some image processing techniques that operate on the intensity component of an image only [17]. An improved alternative to RGB is a perceptual color space such as Hue, Saturation and Voluminous (HSV) that is closer to the human color perception system. The HSV model is based on cylindrical coordinates and it is a nonlinear transformation of the RGB system. HSV space decouples the brightness information from the color information. Thus HSV has one layer for brightness information i.e. $\mathrm{V}$ and two layers for color information i.e. $\mathrm{H}$ and S [17]. It is widely used in artificial vision systems, as it is a powerful tool for the development of digital image processing algorithm based on human color perception model. The HSV model is perfectly suited to portray colors in practical terms for human understanding [18] and is therefore ideal for medical application where the human eye is the ultimate diagnostic tool. Thus here, the scanned foot image is converted into HSV color space, Fig. 1(b), and then a skin classifier is used as a threshold against the image in HSV space. Let $\mathrm{A}$ be the HSV image with dimension $\mathrm{M} \times \mathrm{N}$ and $\mathrm{H}$ be the set of human plantar skin color HSV space

$$
A=\left(\begin{array}{lllll}
\mathrm{a}_{11} & \mathrm{a}_{12} & \mathrm{a}_{13} & \ldots & \mathrm{a}_{1 \mathrm{~N}} \\
\mathrm{a}_{21} & \mathrm{a}_{22} & \mathrm{a}_{23} & \ldots & \mathrm{a}_{2 \mathrm{~N}} \\
& & & \vdots & \\
\mathrm{a}_{(\mathrm{M}-1) 1} & \mathrm{a}_{(\mathrm{M}-1) 2} & \mathrm{a}_{(\mathrm{M}-1) 3} & \ldots & \mathrm{a}_{(\mathrm{M}-1) \mathrm{N}} \\
\mathrm{a}_{\mathrm{M} 1} & \mathrm{a}_{\mathrm{M} 2} & \mathrm{a}_{\mathrm{M} 3} & \ldots & \mathrm{a}_{\mathrm{MN}}
\end{array}\right\}_{\mathrm{M}_{\times} \mathrm{N}}
$$

$\mathrm{H}=\{\mathrm{x} \mid \forall \mathrm{x} \in$ human color skin tone, $\mathrm{x} \in \mathrm{Z}\}$

Using the selected threshold, an object (foot) image is extracted from the background image and yields a binary image, where the white area shows the entire foot and black is the foreground as shown in Fig. 1(c). If $f(\mathrm{x})$ is a function given as

$$
f(\mathrm{x})= \begin{cases}1 & \mathrm{H}_{\min } \leq \mathrm{x} \leq \mathrm{H}_{\max } \\ 0 & \text { otherwise }\end{cases}
$$

Where $\mathrm{H}_{\min }$ and $\mathrm{H}_{\max }$ is the minimum and maximum values of $\mathrm{H}$ and serve as lower and upper threshold bounds respectively. Any pixel of HSV image A, shown as a matrix $\mathrm{A}$, that lies within the human skin color space $\mathrm{H}$ is considered the object (foot) pixels.

$$
\mathrm{B}=\left(\begin{array}{lllll}
\mathrm{b}_{11} & \mathrm{~b}_{12} & \mathrm{~b}_{13} & \ldots & \mathrm{b}_{1 \mathrm{~N}} \\
\mathrm{~b}_{21} & \mathrm{~b}_{22} & \mathrm{~b}_{23} & \ldots & \mathrm{b}_{2 \mathrm{~N}} \\
& & & \vdots & \\
\mathrm{b}_{(\mathrm{M}-1) 1} & \mathrm{~b}_{(\mathrm{M}-1) 2} & \mathrm{~b}_{(\mathrm{M}-1) 3} & \ldots & \mathrm{b}_{(\mathrm{M}-1) \mathrm{N}} \\
\mathrm{b}_{\mathrm{M} 1} & \mathrm{~b}_{\mathrm{M} 2} & \mathrm{~b}_{\mathrm{M} 3} & \ldots & \mathrm{b}_{\mathrm{MN}}
\end{array}\right)_{\mathrm{M} \times \mathrm{N}}
$$

Otherwise, the pixels belong to the background and set to black in color. The HSV image A under $f(\mathrm{x})$ is a binary image and can be represented by a matrix $B$ with dimension $M \times N$ as shown above. Each $b_{i, j}$ has value either 0 or 1 where $\mathrm{i}=0,1,2, \ldots, \mathrm{M}$ and $\mathrm{j}=0,1,2, \ldots, \mathrm{N}$. The element of $\mathrm{B}$ with value 1 is the part of the plantar surface. Let $\mathrm{F}$ be the set that contains all points belonging to plantar surface or the image foreground or more precisely $\mathrm{F}$ contains all white color pixels.

$\mathrm{F}=\{\mathrm{p}(\mathrm{x}, \mathrm{y}) \mid \forall f(\mathrm{p})=1 \Lambda \mathrm{p}(\mathrm{x}, \mathrm{y}) \in \mathrm{B}\}$

Let $\mathrm{S}$ is the set that contains foot stopper color values in HSV space.

$\mathrm{S}=\{\mathrm{x} \mid \forall \mathrm{x} \in$ foot stopper color, $\mathrm{x} \in \mathrm{z}\}$

$$
g(x)= \begin{cases}1, & x \in s \\ 0, & \text { otherwise }\end{cases}
$$

The HSV image A under $\mathrm{g}(\mathrm{x})$ gives the exact foot stopper reference point FS ( $\left.x^{\prime}, y^{\prime}\right)$.

Subsequently, a contour is drawn around the plantar surface and is then bounded by a rectangle called the foot rectangle. The bounded rectangle encloses the entire foot, representing the foot dimension in terms of width and length as shown in Fig. 1(d). The total foot length including toe can be identified by the total length of the rectangle bounding the entire foot.

The Cartesian plane used in the image processing is shown in Fig. 2(a). The origin of Cartesian plane in the image processing lies at top left corner of the image. Thus, the $\mathrm{x}$-axis increases from left to right as usual, while the y-axis increases from the top of the foot to the bottom as indicated in Fig. 2(a). Subsequently, the extreme points along the $\mathrm{x}$-axis of the contours define the width of the rectangle. The region of interest (ROI) for the toe pressure area is bounded by a subrectangle, shown by dotted line in Fig. 1(d), created mathematically using FS point and top left corner point of the original foot rectangle area. The subsequent algorithm then considers only those contour points of the toe that lie within this sub-rectangle and this is explained subsequently. A set T represents all the points in Toe pressure region.

$\mathrm{T}=\left\{\mathrm{p}(\mathrm{x}, \mathrm{y}) \mid \forall \mathrm{y} \leq \mathrm{y}^{\prime} \wedge f(\mathrm{p})=1\right\}$

A central point of the toe polygon of set $\mathrm{T}$ is obtained by Applying trapezoidal centroid as follows

$$
\begin{aligned}
& T_{x}=\frac{1}{6 A_{\mathrm{r}}} \sum_{i=0}^{N-1}\left(X_{i}+X_{i+1}\right)\left(X_{i} Y_{i+1}-X_{i+1} Y_{i}\right) \\
& T_{y}=\frac{1}{6 A_{T}} \sum_{i=0}^{N-1}\left(Y_{i}+Y_{i+1}\right)\left(X_{i} Y_{i+1}-X_{i+1} Y_{i}\right)
\end{aligned}
$$

Where $A_{T}$ is the area of the polygon comprises with the points of $\mathrm{T}$. $\mathrm{N}$ is the cardinality of the set $\mathrm{T}$ or more specifically $\mathrm{N}$ is the number of vertices in the polygon. Subsequently the pressure point of the toe region is given as

$T^{\prime}\left(T_{x}, T_{y}\right)$ 
Then, we must discover the metatarsal area in order to correctly identify the pressure points for this region. Pressure points at edges of the metatarsal area normally lie at the first and fifth metatarsophalangeal joint [6]. Generally the metatarsal area is the widest area of the plantar surface but this is not the case for all feet. Feet are generally classified in two shapes, V-shape and U-shape [10]. The ratio of ball width (distance from first metatarsophalangeal joint to fifth metatarsal joint) and heel width, as shown in Fig. 2(b), changes from person to person. A large value of the ratio indicates a $\mathrm{V}$-shape foot, while a small value indicates a Ushape foot [10]. The only difference between $\mathrm{U}$ and $\mathrm{V}$ shape foot is that the widest part of the foot, in a V-shaped foot always lies in the metatarsal region.

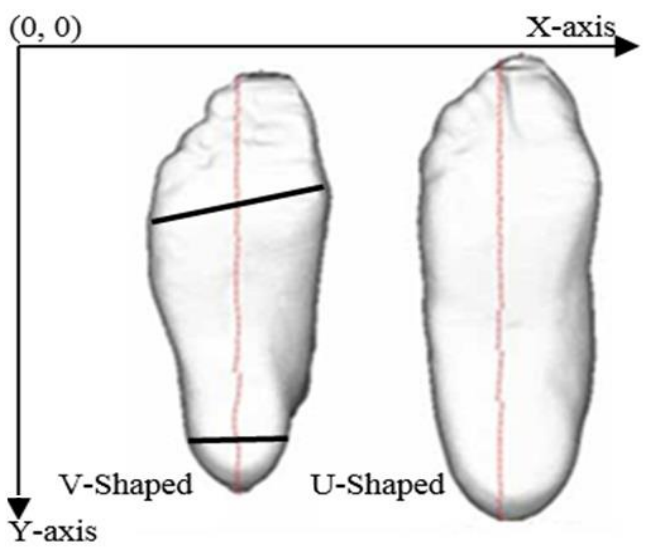

(a)

(b)

Fig. 2. (a) Image plane. (b) $\mathrm{V}$ and $\mathrm{U}$ shaped foot [8]

Subsequently in a V-shape foot the extreme edges along $\mathrm{x}$ axis are the metatarsophalangeal joint, first metatarsal joint at one side and fifth metatarsal joint on the other. In the case of a U-shaped foot, the metatarsal area is first defined as the widest part of the plantar surface may not be the actual metatarsal region. To address these possible errors with such feet, the metatarsal pressure points are ascertained by using the process of foot anthropometry and the contour's extreme points along the $\mathrm{x}$-axis and the FS. To aid algorithm speed, the same process takes place for both $\mathrm{V}$ and $\mathrm{U}$ shaped feet. As the metatarsal area lies in the forefoot area minus the toe, any extreme $\mathrm{x}$-axis points lying above and below the forefoot area are excluded for metatarsal pressure point search. The metatarsal ROI algorithm produces an area bounded by two dotted lines, as shown in Fig. 3. The lower boundary is the line dividing the entire plantar surface foot rectangle in two, perpendicular to the y-axis, while the upper boundary is the line that passes through the FS point and again perpendicular to that point on the y-axis across the plantar surface. The area extracted between the bounded lines is qualified for the metatarsal pressure points search. For pressure points at metatarsal area a subset $\mathrm{M}$ of $\mathrm{F}$ is considered i.e. $\mathrm{M} \subset \mathrm{F}$

$\mathrm{M}=\left\{\mathrm{p}(\mathrm{x}, \mathrm{y}) \mid \forall \mathrm{y} \geq \mathrm{y}^{\prime} \Lambda \mathrm{y} \leq \frac{\max \mathrm{p}(\mathrm{y})+\min \mathrm{p}(\mathrm{y})}{2} \Lambda \mathrm{p}(\mathrm{x}, \mathrm{y}) \in \mathrm{F}\right\}$

Where, $\max p(y)$ is the highest position in vertical direction (Y-axis) in F. Similarly the min $\mathrm{p}(\mathrm{y})$ is the lowest position vertically (Y-axis) in the set $\mathrm{F}$.
Once the metatarsal area is defined, the extreme points of contours are found by running the algorithm on the selected metatarsal area. The extreme points along the $\mathrm{x}$-axis represent the first and fifth metatarsal pressure points that are associated with the metatarsal pressure points region.

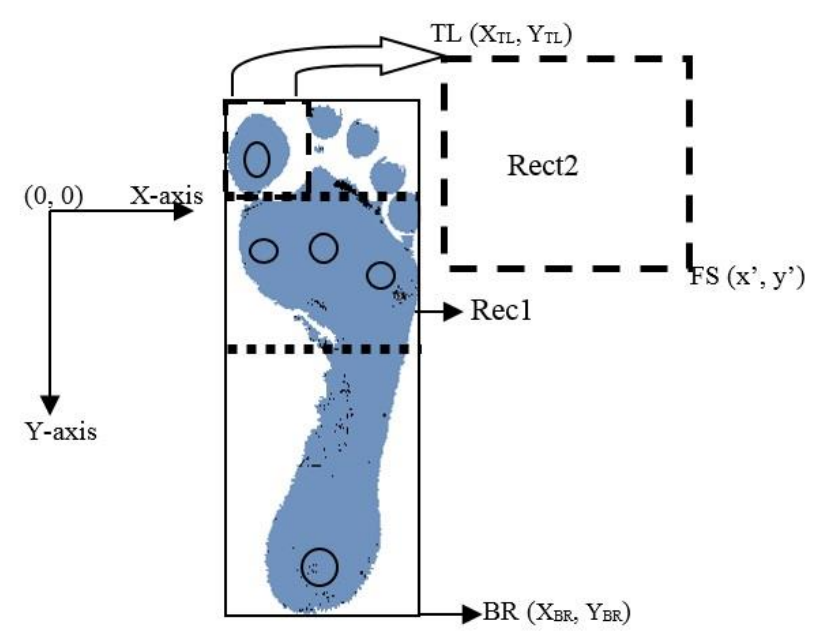

Fig. 3. Pressure points extraction using anthropometry of plantar surface.

The required pressure points at metatarsal are given as;

$\mathrm{M}_{1}\left(\mathrm{M}_{\mathrm{x} 1}, \mathrm{M}_{\mathrm{y} 1}\right) \mid \mathrm{M}_{\mathrm{x} 1}=\min (\mathrm{x}) \wedge \mathrm{M}_{1} \in \mathrm{M}$

$\mathrm{M}_{2}\left(\mathrm{M}_{\mathrm{x} 2}, \mathrm{M}_{\mathrm{y} 2}\right) \mid \mathrm{M}_{\mathrm{x} 2}=\max (\mathrm{x}) \Lambda \mathrm{M}_{2} \in \mathrm{M}$

$\mathrm{M}_{3}\left(\mathrm{M}_{\mathrm{x} 3}, \mathrm{M}_{\mathrm{y} 3}\right) \mid\left(\mathrm{M}_{1}\left(\mathrm{M}_{\mathrm{x} 1}, \mathrm{M}_{\mathrm{y} 1}\right)+\mathrm{M}_{2}\left(\mathrm{M}_{\mathrm{x} 2}, \mathrm{M}_{\mathrm{y} 2}\right)\right) / 2 \Lambda \mathrm{M}_{3} \in \mathrm{M}$

where M1, M2 and M3 are left, right, and middle metatarsal pressure points.

The pressure point at heel is a point in Set F with largest $\mathrm{y}$ coordinate

$H(x, y) \mid y=\max H(y) \in F$

Hence 7, 9, 10,11 and 12 are the extracted pressure points i.e.

$T^{\prime}\left(T_{x}, T_{y}\right), M_{1}\left(M_{x 1}, M_{y 1}\right), M_{2}\left(M_{x 2}, M_{y 2}\right), M_{3}\left(M_{x 3}, M_{y 3}\right)$,

$\mathrm{H}\left(\mathrm{H}_{\mathrm{x}}, \mathrm{H}_{\mathrm{y}}\right)$

The following algorithm shows the step-by-step procedure to find the required pressure points from the ROI's previously described. Those specific regions namely toe (hallux), metatarsal heads and heel (calcaneum).

1. Obtain the foot dimension in terms of length and width using the bounded rectangle Rec1.

2. Obtain the toe ROI by drawing a rectangle Rec2 mathematically using FS(x', y') and the rectangle top left point $\mathrm{TL}\left(\mathrm{X}_{\mathrm{TL}}, \mathrm{Y}_{\mathrm{TL}}\right)$ as shown in Fig. 3.

3. Draw the polygon from the toe contour points bounded by Rec2.

4. Obtain the central point of the toe contour using a trapezoidal centroid.

5. The metatarsal region is ascertained using the foot anthropometry. Once the ROI for the metatarsal is framed, a leftmost point along the $\mathrm{x}$-axis is obtained as a left metatarsal point. Similarly, the right most 
point at metatarsal region is assured by discovering the point with the largest $\mathrm{x}$-coordinate of the contour.

6. Heel pressure point can be identified by finding the point with the largest y-coordinate in contours.

To test the systems accuracy a qualified podiatrist with over fifteen years' experience collaborated to compare the automated and manual SWM pressure detection methods. Foot images from all 70 subjects were provided and the podiatrist independently marked what she identified as the five pressure points on each foot. She then drew a "circle of acceptance" with a diameter of $1 \mathrm{~cm}$ bounding those points and each image was stored separately from the automated findings. A success was considered when the output of the machine identified the same area (anywhere inside the podiatrists drawn circle), a failure if not.

\section{RESULTS}

A group of healthy subjects (44 male and 26 female participants with a mean age of $32.85( \pm 7.4)$ years $)$ were selected. The database consists of 70 images of different ethnicities, age groups and gender, as given below.

\begin{tabular}{|l|c||l|c|}
\hline Age Group & Quantity & Ethnicity & Quantity \\
\hline $20-25$ & 5 & Western European & 15 \\
\hline $25-30$ & 11 & Eastern European & 11 \\
\hline $30-35$ & 16 & African & 15 \\
\hline $35-40$ & 25 & Asian & 20 \\
\hline $40-50$ & 8 & Chinese & 9 \\
\hline $50-70$ & 5 & Total & 70 \\
\hline
\end{tabular}

Table 1: Database age group Table 2: Database Ethnicities

In the first phase, the algorithm initially showed $96 \%$ agreement with the pressure points chosen by the podiatrist. Issues causing the $4 \%$ failure rates were identified and are presented.

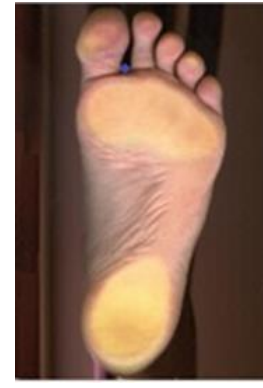

(a)

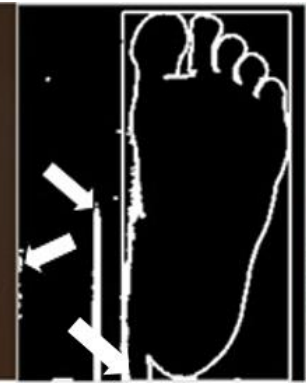

(b)

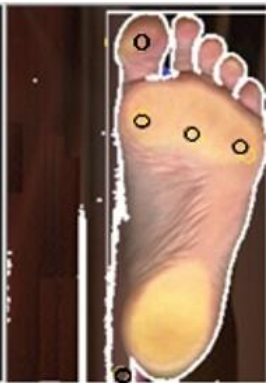

(c)
Fig. 4. Spots in the background similar to skin tone. (a): Input image. (b) Contours of detected plantar surface and false positive detected object in the background (c): Superimpose of foot image and detected foot.

The primary failure occurs when the image processing algorithm mistakenly detects a portion of background as a part of the object (foot), e.g. if a skin tone exists in the background, as shown in the Fig. 4(a). The actual image and the detected edges are superimposed to help the reader visualize the unwanted background as part of the foreground (foot) as shown in Fig. 4(c). A controlled background mitigates such false positive detection.

This controlled background was achieved by the use of a short range optical flat-bed scanner and reduced external lighting conditions. Failure may also occur if skin, other than foot plantar surface, is exposed to the scanner, as shown in Fig. 5. The algorithm extenuates minor patches in the background similar to plantar skin color by Gaussian blur filtering and only considers the single large patch of plantar skin in the image.

This constraint is avoided by ensuring foot placement is performed in a straight and upright manner. This is now ensured by a voice activated system explaining to the user how to place their foot correctly on the scanner and a 5 second sub-scan test performed.

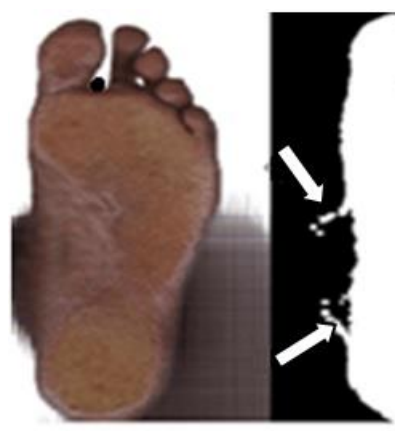

(a)

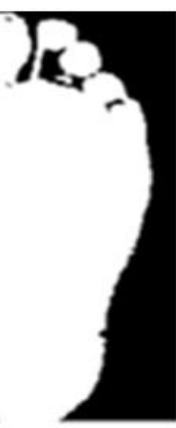

(b)

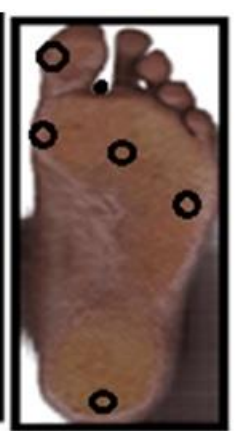

(c)
Fig. 5. Unwanted skin detection proximal to the plantar surface. (a): Input image. (b): Binary image of detected plantar and skin other than plantar surface (c): Incorrect detected pressure points

A final cause of failure is the object, the foot in this case, appearing as separated patches in the binary image, rooted from poor light. Consider Fig. 6, where the toe and other foot digits, numbered by 1,2, 3 and 4, appear separated from the rest of the foot image i.e. patch 5 .
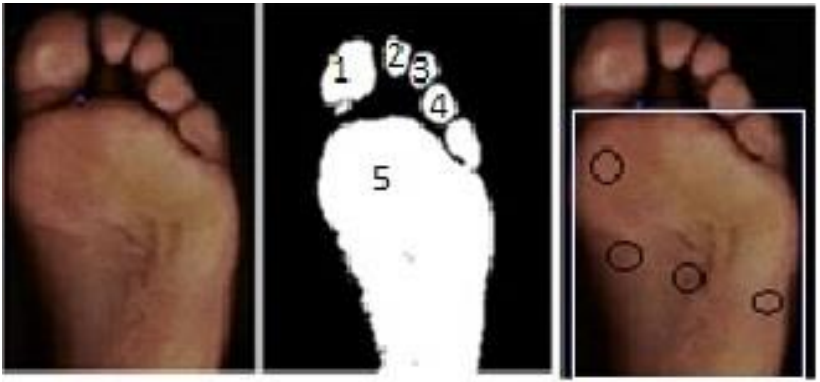

Fig. 6. Object's (foot) patches are numbered in binary image

The 5th patch is the largest patch and comprises a major portion of the foot, while the rest of the patches are the separated foot digits. The rectangle only bounds the largest patch that represents the foot.

The combination of a dilation followed by erosion morphological operations in image processing, known as 'closing', can be used to connect objects in a binary image that are close to each other, or to fill the gaps in the object by using a structure element [19].

The operation is controlled by a structure element which is used to "smooth" the regional boundaries without significant 
or obvious changes to the area [20]. If I is a binary image and $E$ is a structure element, then

$\mathrm{I} \bullet \mathrm{E}=(\mathrm{I} \oplus \mathrm{E}) \ominus \mathrm{E}$

Where $\Theta$ and $\theta$ denote dilation and erosion respectively. Post-closing operation reduced the number of patches without deteriorating the actual size of the foot as shown in Fig. 7.

The proposed solutions above were implemented in a second test phase with the same participants and procedures as before and a $100 \%$ success rate was achieved.
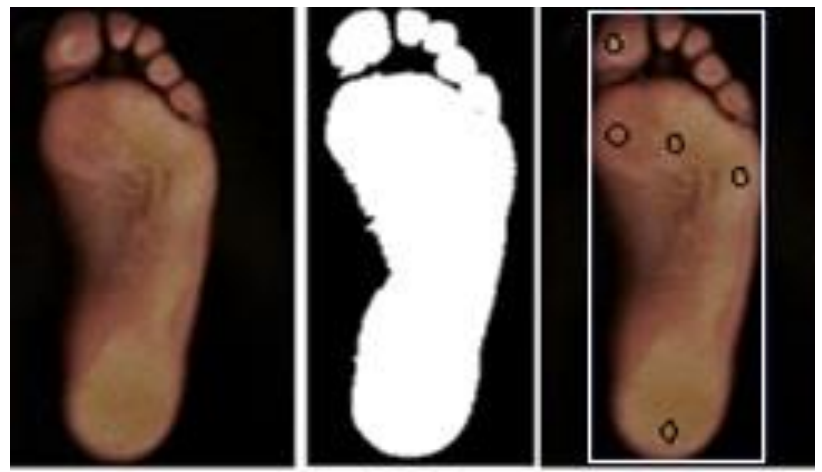

Fig. 7. After dilation with visible contour boundary

Manual and automated testing time comparisons were also evaluated. This evaluation included pressure point detection, manual reporting (original SWME) and result storage. The automated system proposed takes a total $47 \mathrm{~s}$ per foot including data storage and email transmission. The manual SWME process takes in total 180s per foot between viewing a foot, making a pressure point decision, final data handling and storage. This does not include SWM application in either case. This clearly shows the time and information advantages of such an automated approach.

Future research will focus on (a) adding the developed algorithm directly to the physical system architecture and (b) more complex image processing mechanisms to substitute the foot stop with an embedded left and right large toe groove in the perforated sheet. In parallel with the above, lesion recognition on the plantar surface will be performed to avoid subsequent probe application on pressure points where they may overlap a lesion. The authors will consider $3 \mathrm{G}$ system options to account for broadband and wireless fidelity (Wi-Fi) restrictions in developing countries, making use of mobile phone and tablet systems at practitioner sites.

\section{CONCLUSION}

For the first time as far as the authors are aware, an automated pressure point detection system for PSN assessment of a diabetic patient's plantar surface using an optical imaging and foot anthropometry process is presented. The proposed method performs a thresholding process and bounds the foot image in a rectangle followed by contours. Plantar pressure points are then obtained using a combined approach of optical imaging over the HSV three-dimensional color space and plantar anthropometry. The algorithm was successfully applied on 70 participants with a $100 \%$ success result, regardless of the patient's race, age or gender.

\section{ACKNOWLEDGMENT}

Hafeez U. R. Siddiqui is part-sponsored through a London South Bank University PhD Scholarship under the supervision of Sandra Dudley.

\section{REFERENCES}

[1] NHS (2012, November 6th). "Failings in diabetes care cost thousands of lives,".nhs.co.uk. http://www.nhs.uk/news/2012/11November/Pages/ Failings-in-diabetes-care-cost-thousands-of-lives.aspx

[2] N. Hex et al., "Estimating the current and future costs of Type 1 and Type 2 diabetes in the UK, including direct health costs and indirect societal and productivity costs," Diabetic Medicine, vol. 29(7), pp. 855$863,2012$.

[3] S. R. Shah and K.M. Patil, "Processing of foot pressure images and display of an advanced clinical parameter PR in Diabetic Neuropathy," in Proc. 2nd Int. Conf. IEEE EMBS-NE, Arlington, VA, 2005, pp. 5659.

[4] C. Hile, and A. Veves, "Diabetic neuropathy and microcirculation," Current diabetes reports, vol. 3(6), pp. 446-451, 2003.

[5] M. Bharara et al., "Thermography and thermo-metry in the assessment of diabetic neuropathic foot: a case for furthering the role of thermal techniques," The Int. Jour. of lower extremity wounds. vol. 5 (4), pp.250-260, 2006.

[6] NICE (2014, April). "Pressure Ulcers: Prevention and management of pressure ulcers,".nice.org.uk. http://www.nice.org.uk/guidance/cg179 resources/guidance-pressure-ulcers-prevention-and-management-ofpressure-ulcers-pdf

[7] G. M. Bove, "Mechanical sensory threshold testing using nylon monofilaments: The pain field's 'Tin Standard'," Int. Assoc. for the study of pain. vol. 124, pp. 13-17, 2006.

[8] A. Agić et al., "Foot anthropometry and morphology phenomena," Collegium Antropologicum, vol. 30(4), pp. 815-821, 2006.

[9] F. T. Cheng, D. B. Perng, "A systematic approach for developing a foot size information system for shoe last design," Int. Jour. of Industrial Ergonomics, vol. 25(2), pp. 171-185, 1999.

[10] T. G. McPoil et al., "Can foot anthropometric measurements predict dynamic plantar surface contact area?," Jour. of foot and ankle research, vol. 2(1), 2009.

[11] B. Y. S. Tsung et al., "Quantitative comparison of plantar foot shapes under different weight-bearing conditions," Journal of Rehabilitation Research \& Development (JRRD), vol. 40(6), pp. 517-526, 2003.

[12] S. C. Cobb et al., "A Digital Photographic Measurement Method for Quantifying Foot Posture: Validity, Reliability, and Descriptive Data," Jour. of athletic training, vol. 46(1), pp 20-30, 2011.

[13] T. G. McPoil et al., "Arch height change during sit-to-stand: an alternative for the navicular drop test," Jour. of foot and ankle research, pp. vol. 3, 2009.

[14] J. A. Ali et al., "A dense surface modeling technique for foot surface imaging," in Proc. SSSIBI, Wellington, New Zealand, 2011, pp. 295302 .

[15] H. Siddiqui et al., "Automated Peripheral Neuropathy Assessment of Diabetic Patients using Optical Imaging and Binary Processing Techniques," in Proc. IEEE PHT, Bangalore, India, 2013, pp. 200-203.

[16] W. Burger and M. J. Burge, "Color Images," in Digital Image Processing: An Algorithmic Introduction Using Java, 1st Ed., vol. 1, New York: Springer, 2008, ch, 12, pp. 239-305.

[17] P. Sebastian et al., "The Effect of Color Space on Tracking Robustness," in Proc. 3rd Conf. IEEE Indust. Elec. Appl., Singapore, 2008, pp. 25122516.

[18] A. C. Frery and T. Perciano, "Image Data Formats and Color representation," in Introduction to Image Processing Using R, 1st Ed., vol. 1, New York: Springer, 2013, pp. 22.

[19] B. S. D. Sagar, "Mathematical Morphology: An Introduction," in Mathematical morphology in Geomorphology and GISci, 1st Ed. vol. 1, New York: Chapman \& Hall/CR, 2013, pp. 32.

[20] N. Habili et al., "Segmentation of the Face and Hands in Sign Language Video Sequences Using Color and Motion Cues," IEEE Trans. on Circuits and Sys. for Video Technology, vol. 14(8), pp. 1086-109, 2004. 


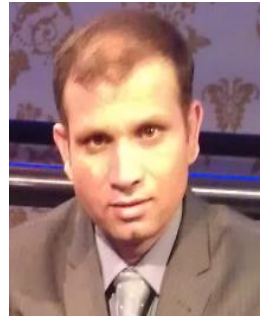

Hafeez-U. R. Siddiqui (S'15) is an Electronic Engineering Ph.D student at London South Bank University. He received the BSc. Degree in Mathematics from Islamia University Bahawalpur (IUB), Pakistan in 1998 and was awarded the MSc. in Computer Science from Bahauddin Zakariya University (BZU) Multan, Pakistan in 2000. He served as a lecturer of computer science in Network Institute of Computer Education (NICE) from 20012006. In 2009 he obtained Master degree in Telecommunications and Computer Network Engineering from London South Bank University London, UK. His research interests include biomedical engineering applications, data recognition, image processing, system embedded programming and machine learning.

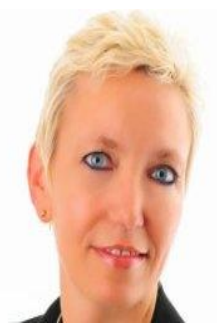

Michelle Spruce FFPM RCPS (Glasg). After qualifying from the University of Southampton with a first-class honours degree in Podiatry, Michelle was seconded to the Cardiovascular Division, Kings College London to complete her doctoral studies. Her work was the first to study the microcirculatory link between capillary occlusion and the development of diabetic neuropathic ulceration. Excerpts of her Ph.D. thesis were presented at both national and international conferences. As a Research Fellow at London South Bank University, Michelle was the first Podiatrist to be elected onto the NHS London Steering Group, where she secured public funding for the training of Clinical Technologists to work with lower limb amputees returning from the UK's conflict zones. Given her academic standing, Michelle strives to promote Podiatry and its educational opportunities in the BRIC countries and throughout Europe under the ERASMUS programme. Meanwhile, she continues to speak at national and international conferences, forever championing the need for patient empowerment and technological advance in the management of diabetic complications.

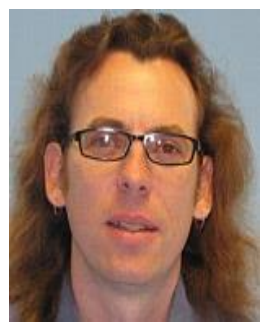

Stephen R. Alty (SM'11 and M'00) received the B.Eng. degree (Hons.) in Electronic Engineering from the University of Liverpool, U.K., in 1992 and the Ph.D. degree in signal processing from Liverpool John Moores University, U.K., in $1998 . \mathrm{He}$ subsequently went on to become a Lecturer with Liverpool John Moores University. Then from 2002, he was a Lecturer in the Division of Engineering at King's College London, London, U.K. Since 2011, he was appointed as a Senior Lecturer with the School of Engineering, London South Bank University, London, U.K. His research interests include adaptive signal processing and machine learning with applications in biomedical engineering and pattern recognition. Dr. Alty is a senior member of the IEEE and a subscriber to the signal processing society (SPS) and engineering in medicine \& biology society (EMBS).

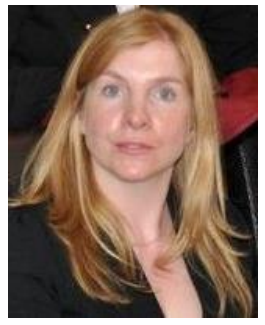

Sandra Dudley (M'12) was born in Carlow, the Republic of Ireland. She received the BSc (Hons) and $\mathrm{Ph} . \mathrm{D}$ Degrees in Physics from the University of Essex, Essex. U.K. in 1998 and 2004 respectively. Subsequently, she spent two years working as a postdoctoral researcher at Essex University on a British Telecom project resulting in world record broadband-system power consumption for last mile access. Since August 2009 she joined the School of Engineering, London South Bank University, London, U.K. as a lecturer and become a Principal lecturer in March 2014. Her research interests are in physical layer system design, automated health technologies, data recognition, optical-wireless systems and solution finding for the broadband urban-rural divide. Dr. Dudley is a member of the IEEE and an IEEE journal reviewer. 